\title{
Inner-Outer Factorization for Nonlinear Noninvertible Systems
}

\author{
Joseph A. Ball, Mark A. Petersen, and Arjan van der Schaft
}

\begin{abstract}
This paper considers inner-outer factorization of asymptotically stable nonlinear state space systems in continuous time that are noninvertible. Our approach will be via a nonlinear analogue of spectral factorization which concentrates on first finding the outer factor instead of the inner factor. An application of the main result to control of nonminimum phase nonlinear systems is indicated.
\end{abstract}

Index Terms-Hamilton-Jacobi inequality, inner-outer factorization, nonlinear noninvertible systems, nonlinear Smith predictor.

\section{INTRODUCTION}

$\mathrm{C}$ ONSIDER a smooth nonlinear system of the form (in local coordinates $x=\left(x_{1}, \ldots, x_{n}\right)$ for the $n$-dimensional statespace manifold $\mathcal{M}$ )

$$
\Sigma: \begin{cases}\dot{x}=a(x)+b(x) u, & u \in \mathbb{R}^{m} \\ y=c(x)+d(x) u, & y \in \mathbb{R}^{p}\end{cases}
$$

where $a(x)$ and the columns of the matrix $b(x)$ are smooth vector fields on $\mathcal{M}$. Furthermore, $c: \mathcal{M} \rightarrow \mathbb{R}^{p}$ and $d: \mathcal{M} \rightarrow$ $\mathbb{R}^{p \times m}$ are smooth mappings (at least $C^{1}$ ). Throughout, we assume that there exists an equilibrium $x_{0}$ for $u=0$, that is, $a\left(x_{0}\right)=0$, while $c\left(x_{0}\right)=0$.

The Hamiltonian extension (see [15]) of $\Sigma$ has the form

$$
\left\{\begin{array}{l}
\dot{x}=a(x)+b(x) u \\
\dot{p}=-\left[\frac{\partial a}{\partial x}(x)+\frac{\partial b}{\partial x}(x) u\right]^{T} p-\frac{\partial^{T} c}{\partial x}(x) u_{a}-u^{T} \frac{\partial^{T} d}{\partial x}(x) u_{a} \\
y=c(x)+d(x) u \\
y_{a}=b^{T}(x) p+d^{T}(x) u_{a}
\end{array}\right.
$$

where $u, y_{a} \in \mathbb{R}^{m}$ and $u_{a} \in \mathbb{R}^{p}$. Imposing the interconnection law $u_{a}=y$ in (2), we obtain the Hamiltonian system

$$
[D \Sigma]^{T} \circ \Sigma:\left\{\begin{array}{l}
\dot{x}=\frac{\partial H}{\partial p}(x, p, u) \\
\dot{p}=-\frac{\partial H}{\partial x}(x, p, u) \\
y_{a}=\frac{\partial H}{\partial u}(x, p, u)
\end{array}\right.
$$

Manuscript received October 10, 2002; revised June 10, 2003. Recommended by Associate Editor R. Freeman.

J. A. Ball is with the Department of Mathematics, Virginia Polytechnic Institute and State University, Blacksburg, VA 24061-0123 USA (e-mail: ball@math.vt.edu).

M. A. Petersen is with the Department of Mathematics, Potchefstroom University for CHE, Potchefstroom x6001, South Africa (e-mail: wskmap@puknet.puk.ac.za).

A. J. van der Schaft is with the Department of Applied Mathematics, University of Twente, 7500AE Enschede, The Netherlands (e-mail: a.j.vanderschaft@math.utwente.nl).

Digital Object Identifier 10.1109/TAC.2004.825644 with Hamiltonian function $H(x, p, u)$ given by

$$
\begin{aligned}
H(x, p, u)=p^{T}[a(x) & +b(x) u] \\
& +\frac{1}{2}[c(x)+d(x) u]^{T}[c(x)+d(x) u] .
\end{aligned}
$$

Here, the state-space is the cotangent bundle $T^{*} \mathcal{M}$ of the state manifold $\mathcal{M}$ for $\Sigma$, inputs $u$ are from $\mathbb{R}^{m}$, while also the outputs $y_{a}$ are from $\mathbb{R}^{m}$. Furthermore, $D \Sigma$ denotes the Fréchet derivative of the input-output system $\Sigma$ (see [11] and [15] for details and notation).

We view the system decomposition $[D \Sigma]^{T} \circ \Sigma$ as a nonlinear analogue of a spectral factorization. We observe that in the linear setting, $[D \Sigma]^{T} \circ \Sigma$ reduces to the series interconnection of $\Sigma$ and its adjoint linear system $\Sigma^{*}$, having transfer matrix $G^{T}(-s) G(s)$ if $G(s)$ is the transfer matrix of $\Sigma$.

On the other hand, a nonlinear analogue of inner-outer factorization may be given as follows (see [3], [5], [8], [11], and [44] for further information). A nonlinear system $\Theta$ as in (1) (with inputs $\bar{y}$ and outputs $y$ )

$$
\Theta:\left\{\begin{array}{l}
\dot{x}=a_{\Theta}(x)+b_{\Theta}(x) \bar{y} \\
y=c_{\Theta}(x)+d_{\Theta}(x) \bar{y}
\end{array}\right.
$$

is called inner if there exists a nonnegative-valued storage function $P(x)$ with $P\left(x_{0}\right)=0$ such that

$$
P\left(x\left(t_{2}\right)\right)-P\left(\left(x\left(t_{1}\right)\right)=\frac{1}{2} \int_{t_{1}}^{t_{2}}\left[\|\bar{y}(t)\|^{2}-\|y(t)\|^{2}\right] d t\right.
$$

over all trajectories $(\bar{y}(t), x(t), y(t))$ of the system.

Condition (6) can be expressed equivalently by saying that $\Theta$ is lossless with respect to the $L_{2}$-gain supply rate

$$
s(u, y)=\frac{1}{2} \bar{y}^{T} \bar{y}-\frac{1}{2} y^{T} y .
$$

If $P$ is assumed to be smooth, the energy balance relation (6) can be expressed in infinitesimal form as

$$
\begin{aligned}
P_{x}(x) b_{\Theta}(x)+c_{\Theta}(x)^{T} d_{\Theta}(x) & =0 \\
P_{x}(x) a_{\Theta}(x)+\frac{1}{2} c_{\Theta}(x)^{T} c_{\Theta}(x) & =0 \\
d_{\Theta}(x)^{T} d_{\Theta}(x) & =I .
\end{aligned}
$$

A nonlinear outer system may be defined as follows. Let $\Sigma$ be a system as in (1). Then we define $\Sigma^{-1}$ as the system so that $(u, x, y)$ is a trajectory of $\Sigma$ if and only $(y, x, u)$ is a trajectory for $\Sigma^{-1}$ (i.e., the roles of inputs and outputs are interchanged in going from $\Sigma$ to $\Sigma^{-1}$. Under the assumption that $d(x)$ is 
invertible for each $x$, it is easy to derive the input-state-output realization for $\Sigma^{-1}$ :

$$
\Sigma^{-1}:\left\{\begin{array}{l}
\dot{x}=\left(a(x)-b(x) d^{-1}(x) c(x)\right)+b(x) d(x)^{-1} y \\
u=-d(x)^{-1} c(x)+d(x)^{-1} y .
\end{array}\right.
$$

We then say that $\Sigma$ is minimum phase if $x_{0}$ is an asymptotically stable equilibrium of the vector field $a(x)-b(x) d(x)^{-1} c(x)$ (also called the zero dynamics of $\Sigma$ ). If $x_{0}$ is merely a Lyapunov stable equilibrium of $a(x)-b(x) d(x)^{-1} c(x)$ then $\Sigma$ is called weakly minimum phase. An extension of this notion of zero dynamics (or output-nulling dynamics) to the noninvertible case will be given and used in the sequel (see [14] and [26]). If $x_{0}$ is an asymptotically stable equilibrium of $a(x)$ and $\Sigma$ is weakly minimum phase, we say that $\Sigma$ is outer. (Note that the definitions of "inner" and "outer" given above attempt to generalize these notions from the linear case to the nonlinear case. As such there is some flexibility in the definitions; for example, we may want to strengthen the condition of asymptotic stability in the definition of outer to global asympotic stability, or we may want to add conditions on the linearization at $x_{0}$. Also, the current definition of "inner" only implies Lyapunov stability under additional conditions, such as positive definiteness of $P$ at $x_{0}$.)

The nonlinear inner-outer factorization problem for a given input-state-output nonlinear system $\Sigma$ as in (1) can now be formulated as finding an inner system $\Theta$ and an outer system $\Phi$ in state-space form such that

$$
\Sigma=\Theta \circ \Phi
$$

By this, we mean that for every initial condition of the nonlinear system $\Sigma$ there exist initial conditions of $\Theta$ and $\Phi$ such that the input-output behavior for $\Sigma$ equals the input-output behavior of the series interconnection of $\Phi$ followed by $\Theta$. Since, after cancellation of nonobservable/noncontrollable states, $[D \Theta]^{T} \circ \Theta=$ $I$ (the static identity map) if $\Theta$ is inner (see [4]), we see the formal connection between inner-outer and spectral factorization: if $\Phi$ is an outer system for which

$$
[D \Sigma]^{T} \circ \Sigma=[D \Phi]^{T} \circ \Phi
$$

then $\Phi$ is the outer factor for an inner-outer factorization $\Sigma=$ $\Theta \circ \Phi$ of $\Sigma$.

There has already been much work on the nonlinear inner-outer/spectral factorization problem. In a series of papers (see example, [2], [4], and [5]), Ball and Helton investigated inner-outer factorization of nonlinear input-output operators and of nonlinear state space systems in discrete time. This was achieved by constructing an invertible, lossless (inner) system having given zero dynamics in terms of the solution of a certain Hamilton-Jacobi equation. By contrast, in [11] (also, in [8]) a version of the same results was discovered but for stable, invertible, continuous-time systems having state-space equations that are input affine. Specifically, they formulated and solved (in terms of the solution of a Hamilton-Jacobi equation) a nonlinear spectral factorization problem that facilitates the computation of the outer factor in an inner-outer factorization of given stable plants. It is shown how to obtain the outer factor $\Phi$ by "spectral factorization" of the Hamiltonian system
$[D \Sigma]^{T} \circ \Sigma$ under the assumption that $E(x):=d^{T}(x) d(x)$ is invertible for all $x$. In fact, if this invertibility condition is satisfied then we may directly compute the inverse system $\left([D \Sigma]^{T} \circ \Sigma\right)^{-1}$. The outer factor is now obtained by computing the stable invariant manifold of this inverse system via a Hamilton-Jacobi equation. In [8], a description of an inner system as part of an inner-outer factorization was given in terms of the smooth solution of a certain type of Hamilton-Jacobi equation. Here, the system was realized in a manner which proves useful for the extension of the arithmetic of nonlinear inner systems and their factorization. In [39] we make use of the theory of inner-outer factorization to provide a parametrization of nonsquare spectral factors in terms of Lagrangian manifolds and Hamilton-Jacobi equations. Also, the parametrization of nonsquare spectral factors in terms of inner factorizations of inner systems is discussed. Explicit formulas for the systems are determined.

In the linear case, two classes of methods are used to compute inner-outer factorizations for both discrete-time and continuous-time systems. The first can be termed a one-shot approach because it relies on the determination of stabilizing solutions of certain Riccati or generalized Riccati equations. In this approach we first compute a right invertible outer (or spectral) factor and then compute the corresponding inner factor. Our approach for the nonlinear problem can be viewed as the nonlinear analogue of this first approach. The second class does not require one to solve a Riccati equation but instead is based on recursive dislocation of unstable zeros of the transfer function matrix by premultiplying it with suitable elementary all-pass factors. This class is generated by methods where a square inner system is computed before the corresponding outer factor. This recursive zeros dislocation technique has the major advantage that it is usually more numerically reliable and applies for a very general class of factorization problems. However, by using this method, one is not usually able to determine explicit formulas for the inner, outer and spectral factors. We do not know a nonlinear analogue of this second approach.

The main point of the present paper is to extend the results in [10] and [11] on nonlinear spectral factorization for nonlinear input-state-output systems to the case where $E(x)=$ $d^{T}(x) d(x)$ may not be invertible. It is then no extra work to deal with a more general class of smooth input-state-output system not necessarily affine with respect to the input variable as in (1) [see (16)]. As in [10] and [11], our approach will be via nonlinear spectral factorization which concentrates on first determining the outer factor instead of the inner factor. As in [11], this outer factor is found by first finding a change of coordinates $(x, p) \rightarrow(x, \bar{p})$ (with $p=\bar{p}+P_{x}(x)$ for some smooth realvalued function $P(x)$ ) so that the spectral factorization $[D \bar{\Sigma}]^{T}$ 。 $\bar{\Sigma}$ for an outer $\bar{\Sigma}$ is apparent in the new coordinate system. The new idea for the noninvertible case here is to identify the required real-valued function $P(x)$ as the performance (or optimal-value) function for a singular optimal control problem; then we may call upon results from linear singular optimal control (see [1], [17], [21], [33], and [41] to see how to proceed for the nonlinear case. By contrast, in [5], the inner factor was found first as the inner system having the appropriate prescribed zero dynamics. In the end, we construct a lossless system with given 
zero dynamics in terms of the solution of a certain Hamilton-Jacobi inequality (for more on Hamilton-Jacobi inequalities, see [43] and [44]).

In linear as well as nonlinear control theory, inner-outer factorization (or more generally $J$-inner-outer factorization) of rational matrix functions has played an important role. For example, in the theory of $H_{\infty}$-optimal control ([7], [22], [23], [28], [29] for linear; [4]-[6], [9]-[11], [42], [44] for nonlinear) and nonlinear chemical process control ([16], [31], [32], [49]), this type of factorization is used extensively. In our paper, we will mainly discuss nonlinear inner-outer factorization and its application in the former two settings. In particular, in [18]-[20] a function-theoretic approach is adopted and explicit state space formulas for inner systems and corresponding inner-outer factors are found. Furthermore, linear inner-outer factorization and its connection with the linear spectral factorization problem are discussed in ([18]-[20], [24], and [46]). This paper (together with [8], [11], and [37]-[39]) considers some of the nonlinear analogues of these results.

A specific area of application for results of the type to be discussed here is process control. In nonlinear theory it has been asserted that the control design of nonminimum phase (i.e., unstable zero dynamics in the sense of Byrnes-Isidori [13]), stable systems may be based upon the inverse of the minimum phase (outer) factor, with the inner (all-pass) factor remaining as a limiting element in the closed loop system. In particular, in process control, the problem of finding an invertible outer factor has been discussed for minimum phase approximations to single-input-single-output (SISO), nonlinear, nonminimum phase systems in [16], second-order systems in [32] and involutive systems in [49]. In the latter two papers, the strategy involves the derivation of a new nonlinear output map from the original state dynamics which yields the same steady-state locus but which has stable zero dynamics. We touch on this type of application in Section III, and show how a nonlinear version of the Smith predictor may be derived.

\section{INNER-OUTER FACTORIZATION FOR NONLINEAR NONINVERTIBLE SYSTEMS}

Consider (1) under the following standing assumption.

Assumption 1: The equilibrium $x_{0}=0$ of $\dot{x}=a(x)$ is locally exponentially stable, that is, all eigenvalues of $\partial a / \partial x(0)$ are in the open left half-plane. Furthermore, $c(0)=0$.

First, we recall from [11] how to construct an inner-outer factorization $\Sigma=\Theta \circ \bar{\Sigma}$ in the case where $E(x):=d^{T}(x) d(x)$ is invertible. We consider as before (3) the Hamiltonian system

$$
[D \Sigma]^{T} \circ \Sigma:\left\{\begin{array}{l}
\dot{x}=\frac{\partial H}{\partial p}(x, p, u) \\
\dot{p}=-\frac{\partial H}{\partial x}(x, p, u) \\
y_{a}=\frac{\partial H}{\partial u}(x, p, u)
\end{array}\right.
$$

with inputs $u \in \mathbb{R}^{m}$ and outputs $y_{a} \in \mathbb{R}^{m}$, and the Hamiltonian function $H(x, p, u)$ given by

$$
\begin{aligned}
H(x, p, u)=p^{T}[a(x)+b(x) u] & \\
& +\frac{1}{2}[c(x)+d(x) u]^{T}[c(x)+d(x) u] .
\end{aligned}
$$

Recall that the state-space of $[D \Sigma]^{T} \circ \Sigma$ is the cotangent manifold $T^{*} \mathcal{M}$ of the state manifold $\mathcal{M}$ for $\Sigma$. Under the assumption that $E(x)$ is invertible for each $x$, we may invert $[D \Sigma]^{T} \circ \Sigma$ (i.e., interchange the roles of the input $u$ and the output $y$ ) to come up with another Hamiltonian system

$$
\left([D \Sigma]^{T} \circ \Sigma\right)^{-1}:\left\{\begin{array}{l}
\dot{x}=\frac{\partial H^{\times}}{\partial p}\left(x, p, y_{a}\right) \\
\dot{p}=-\frac{\partial H^{\times}}{\partial x}\left(x, p, y_{a}\right) \\
u=\frac{\partial H^{\times}}{\partial y_{a}}\left(x, p, y_{a}\right)
\end{array}\right.
$$

with state-space $T^{*} \mathcal{M}$, inputs $y_{a}$ and outputs $u$, where the inverse Hamiltonian $H^{\times}$is the Legendre transform of $H(x, p, u)$ with respect to $u$ and $y_{a}$, i.e.,

$$
\begin{aligned}
H^{\times}\left(x, p, y_{a}\right) & =H(x, p, u)-u^{T} y_{a} \\
u \text { satisfying } \frac{\partial H}{\partial u}(x, p, u) & =y_{a} .
\end{aligned}
$$

Explicitly, this works out to be

$$
\begin{aligned}
H^{\times}\left(x, p, y_{a}\right)= & p^{T}\left(a(x)-b(x) E^{-1}(x) d^{T}(x) c(x)\right) \\
& +\frac{1}{2} c^{T}(x)\left(I-d(x) E^{-1}(x) d^{T}(x)\right) c(x) \\
& -\frac{1}{2} p^{T} b(x) E^{-1}(x) b^{T}(x) p \\
& +\left(p^{T} b(x)+c^{T}(x) d(x)\right) E^{-1}(x) y_{a} \\
& -\frac{1}{2} y_{a}^{T} E^{-1}(x) y_{a}
\end{aligned}
$$

where $E(x)=d^{T}(x) d(x)$. One then defines a canonical change of coordinates $(x, p) \rightarrow(\bar{x}, \bar{p})$ such that $\left.\{\bar{x}, \bar{p}) \in T^{*} \mathcal{M}: \bar{x}=0\right\}$ is an antistable invariant manifold for the Hamiltonian flow induced by $H$ (with $u=0$ ), and the manifold $\left\{(\bar{x}, \bar{p}) \in T^{*} \mathcal{M}: \bar{p}=0\right\}$ is a Lyapunov stable invariant manifold of $H^{\times}$(with $y_{a}=0$ ). By Assumption 1 we may just take $x=\bar{x}$. Indeed, since the matrix $-\partial^{T} a / \partial x(0)$ has all its eigenvalues in the open right half-plane, the manifold $\{(x, \bar{p}): x=0\}$ is invariant and antistable, for all choices of $\bar{p}$. To compute a Lagrangian invariant manifold for $\left([D \Sigma]^{T} \circ \Sigma\right)^{-1}$ with $y_{a}=0$, one then considers the Hamilton-Jacobi equation $H^{\times}\left(x, P_{x}^{T}(x), 0\right)=0$ for some smooth function $P(x)$ with $P(0)=0$, i.e.,

$$
\begin{aligned}
& P_{x}(x)\left[a(x)-b(x) E^{-1}(x) d^{T}(x) c(x)\right] \\
& \quad+\frac{1}{2} c^{T}(x)\left[I-d(x) E^{-1}(x) d^{T}(x)\right] c(x) \\
& \quad-\frac{1}{2} P_{x}(x) b(x) E^{-1}(x) b^{T}(x) P_{x}^{T}(x)=0
\end{aligned}
$$

with the stability side constraint

$$
\begin{aligned}
\frac{\partial H^{\times}}{\partial p}\left(x, P_{x}^{T}(x), 0\right)= & a(x)-b(x) E^{-1}(x) \\
& \times\left[d^{T}(x) c(x)+b^{T}(x) P_{x}^{T}(x)\right] \\
& \text { is Lyapunov stable. }
\end{aligned}
$$

Given a smooth solution $P(x)$ of (12) and (13), then the set of points

$$
\left\{(x, p): p=P_{x}^{T}(x)\right\}
$$


is an invariant manifold of $\left([D \Sigma]^{T} \circ \Sigma\right)^{-1}$ with $y_{a}=0$ on which the dynamics coordinated by $x$ is given by the Lyapunov stable vector field in (13). Then, after the canonical change of coordinates $(x, p) \rightarrow(x, \bar{p})$ with

$$
p=\bar{p}+P_{x}^{T}(x)
$$

the set of points $\{(x, \bar{p}): x=0\}$ remains the antistable invariant manifold for $[D \Sigma]^{T} \circ \Sigma$ with $u=0$, while the invariant manifold (14) for $\left([D \Sigma]^{T} \circ \Sigma\right)^{-1}$ with $y_{a}=0$ is given simply as $\{(x, \bar{p}): \bar{p}=0\}$. In the new coordinates, the original Hamiltonian $H(x, p, u)$ assumes the form

$$
\begin{aligned}
H(x, p, u)= & H\left(x, \bar{p}+P_{x}^{T}((x), u)\right. \\
= & \bar{p}^{T}(a(x)+b(x) u) \\
& +\frac{1}{2}(\bar{c}(x)+\bar{d}(x) u)^{T}(\bar{c}(x)+\bar{d}(x) u) \\
= & : \bar{H}(x, \bar{p}, u)
\end{aligned}
$$

where $\bar{d}(x)$ is chosen to be a smooth, invertible, $m \times m$ solution of

$$
E(x)=\bar{d}^{T}(x) \bar{d}(x) .
$$

It then develops that $[D \Sigma]^{T} \circ \Sigma=[D \bar{\Sigma}]^{T} \circ \bar{\Sigma}$ where $\bar{\Sigma}$ is given by

$$
\bar{\Sigma}:\left\{\begin{array}{l}
\dot{x}=a(x)+b(x) u \\
\bar{y}=\bar{c}(x)+\bar{d}(x) u
\end{array}\right.
$$

where $\bar{d}$ is as in (15) and $\bar{c}$ is given by

$$
\bar{c}(x)=\bar{d}(x) E^{-1}(x)\left[d^{T}(x) c(x)+b^{T}(x) P_{x}^{T}(x)\right] .
$$

Since we are assuming that $a(x)$ is locally exponentially stable with respect to the equilibrium point $x_{0}$, it follows that $\bar{\Sigma}$ is asymptotically stable. The dynamics for $\bar{\Sigma}^{-1}$ (with $\bar{y}=0$ ) works out to be the vector field (13), and hence is Lyapunov stable. Thus, $\Sigma$ is outer, and one can verify the spectral factorization property $[D \Sigma]^{T} \circ \Sigma=[D \bar{\Sigma}]^{T} \circ \bar{\Sigma}$. Under the assumption that $P(x) \geq 0$, one can compute that $\Theta:=\Sigma \circ \bar{\Sigma}^{-1}$ is inner with storage function $P$ (with respect to supply rate $1 / 2 \bar{y}^{T} \bar{y}-1 / 2 y^{T} y$, and we conclude that $\Sigma=\Theta \circ \bar{\Sigma}$ is an inner-outer factorization for $\Sigma$. (We refer to [11], [43] for a discussion about $P(x) \geq 0$ actually being implied by strengthening the stability side constraint (13) to asymptotic stability.)

In case $E(x):=d^{T}(x) d(x)$ is not invertible for all $x$, this procedure breaks down: it is not easy to define the inverse of $[D \Sigma]^{T} \circ \Sigma$ and the Hamilton-Jacobi (12) does not make immediate sense. The clue to how to proceed in the more general case where $E(x)$ is not invertible, however, is provided by the observation that the Hamilton-Jacobi (12) is actually the Hamilton-Jacobi-Bellman equation for the nonlinear optimal control problem of minimizing the cost criterion $1 / 2 \int_{0}^{\infty}\|y(t)\|^{2} d t$. This can be also seen from the fact that the Hamiltonian $H(x, p, u)$ of the Hamiltonian system $[D \Sigma]^{T} \circ \Sigma$ is nothing else than the pre-Hamiltonian of this optimal control problem. In the invertible case $(E(x)$ invertible) this optimal control problem is a regular problem leading to the Hamilton-Jacobi-Bellman (12), while in the general noninvertible case the optimal control problem is singular, leading merely to a dissipation inequality.

Actually it will be notationally simpler in this paper to study the inner-outer factorization problem in the noninvertible case immediately for smooth general systems (see [44])

$$
\Sigma: \begin{cases}\dot{x}=F(x, u), & F(0,0)=0 \\ y=G(x, u), & G(0,0)=0\end{cases}
$$

with $u \in \mathbb{R}^{m}, y \in \mathbb{R}^{p}$, and $x=\left(x_{1}, \ldots, x_{n}\right)$ local coordinates for a state-space manifold $\mathcal{M}$. Then, the Hamiltonian extension for $\Sigma$ is given by

$$
\left\{\begin{array}{l}
\dot{x}=F(x, u) \\
\dot{p}=-\frac{\partial^{T} F}{\partial x}(x, u) p-\frac{\partial^{T} G}{\partial x}(x, u) u_{a} \\
y=G(x, u) \\
y_{a}=\frac{\partial^{T} F}{\partial u}(x, u) p+\frac{\partial^{T} G}{\partial x}(x, u) u_{a}
\end{array}\right.
$$

and imposition of the interconnection law $u_{a}=y$ leads us to the Hamiltonian system

$$
[D \Sigma]^{T} \circ \Sigma:\left\{\begin{array}{l}
\dot{x}=F(x, u) \\
\dot{p}=-\frac{\partial^{T} F}{\partial x}(x, u) p-\frac{\partial^{T} G}{\partial x}(x, u) G(x, u) \\
y_{a}=\frac{\partial^{T} F}{\partial u}(x, u) p+\frac{\partial^{T} G}{\partial u}(x, u) G(x, u)
\end{array}\right.
$$

or, in Hamiltonian form

$$
[D \Sigma]^{T} \circ \Sigma:\left\{\begin{array}{l}
\dot{x}=\frac{\partial H}{\partial p}(x, p, u) \\
\dot{p}=-\frac{\partial H}{\partial x}(x, p, u) \\
y_{a}=\frac{\partial H}{\partial u}(x, p, u)
\end{array}\right.
$$

with Hamiltonian

$$
H(x, p, u)=p^{T} F(x, u)+\frac{1}{2} G^{T}(x, u) G(x, u) .
$$

To produce a system $\bar{\Sigma}$ which solves the spectral factorization problem

$$
[D \Sigma]^{T} \circ \Sigma=[D \bar{\Sigma}]^{T} \circ \bar{\Sigma}
$$

we again look for a change of variable

$$
(x, p) \rightarrow(x, \bar{p}) \text { with } p=\bar{p}+P_{x}^{T}(x)
$$

for some smooth function $P: \mathcal{M} \rightarrow \mathbb{R}$. Then, in the new coordinates the Hamiltonian (20) takes the form

$$
\begin{aligned}
\bar{H}(x, \bar{p}, u)= & H\left(x, \bar{p}+P_{x}^{T}(x), u\right) \\
= & \bar{p}^{T} F(x, u)+P_{x}^{T}(x) F(x, u) \\
& +G(x, u)^{T} G(x, u) .
\end{aligned}
$$

Hence, if we assume that $x \rightarrow P(x)$ is a smooth function on $\mathcal{M}$ satisfying the dissipation inequality

$$
P_{x}(x) F(x, u)+\frac{1}{2} G^{T}(x, u) G(x, u) \geq 0
$$

and that we can find a smooth vector-valued function $(x, u) \rightarrow$ $\bar{G}(x, u)$ satisfying the factorization problem

$$
\bar{G}^{T}(x, u) \bar{G}(x, u)=P_{x}(x) F(x, u)+\frac{1}{2} G^{T}(x, u) G(x, u)
$$


then, by comparing (22) with (20), we see that the system $\bar{\Sigma}$ given by

$$
\bar{\Sigma}:\left\{\begin{array}{l}
\dot{x}=F(x, u) \\
\bar{y}=\bar{G}(x, u)
\end{array}\right.
$$

produces a solution of the nonlinear spectral factorization problem (21). As was done for the invertible case, we take as a standing assumption that

Assumption 2: The eigenvalues of $\partial F / \partial x(0,0)$ are in the open left half-plane.

Hence, any system $\bar{\Sigma}$ of the form (25) is asymptotically stable. The goal now is to construct the function $P(x)$ is such a way to guarantee that the resulting $\bar{\Sigma}$ is outer, i.e., so that in addition $\bar{\Sigma}$ has stable output nulling dynamics.

As explained in the Introduction, for the case of input-affine systems $\Sigma$ as in (1), we define the output-nulling dynamics (or zero dynamics, [14]) for a general system $\Sigma$ as in (16) to be the set of all state trajectories $x(\cdot)$ generated by some input trajectory $u(\cdot)$ such that $y(t)=G(x(t), u(t))$ is identically zero. Under some regularity conditions (see [25], [36]) the outputnulling dynamics can be computed as follows. First, we compute the maximal controlled invariant output-nulling submanifold $\mathcal{N}^{*} \subset \mathcal{M}$ (if it exists) as the maximal submanifold $\mathcal{N} \subset \mathcal{M}$ containing $x=0$, for which there exists a smooth feedback $u=\alpha(x), \alpha(0)=0$, such that

$$
\begin{aligned}
& F(x, \alpha(x)) \in T_{x} \mathcal{N} \text { for all } x \in \mathcal{N} \\
& G(x, \alpha(x))=0 \text { for all } x \in \mathcal{N} .
\end{aligned}
$$

In general, such a smooth feedback $\alpha(x)$ is not unique, and (again under some regularity conditions; see, e.g., [36, Ch. 11]) the whole family of feedbacks $u=\alpha(x)$ satisfying (26) for $\mathcal{N}=\mathcal{N}^{*}$ can be parametrized as $\alpha(x, v)$, with $v \in \mathbb{R}^{\tilde{m}}, \widetilde{m} \leq$ $m$. Then, the output-nulling dynamics is generated by the lower dimensional dynamics

$$
\widetilde{\Sigma}: \quad \dot{\widetilde{x}}=\widetilde{F}(\widetilde{x}, v), \quad \widetilde{x} \in \mathcal{N}^{*}, \quad \widetilde{F}(0,0)=0
$$

where $\widetilde{F}(\widetilde{x}, v)=F(\widetilde{x}, \alpha(\widetilde{x}, v))$.

Definition 1: $\Sigma$ in (16) is weakly minimum phase if its output-nulling dynamics $\widetilde{\Sigma}$ (see (27)) can be rendered Lyapunov stable with regard to $x \in \mathcal{N}^{*}$ by a smooth feedback $v=\widetilde{\alpha}(\widetilde{x})$. If the output-nulling dynamics $\widetilde{\Sigma}$ can be rendered locally asymptotically stable, then $\Sigma$ is called minimum phase

Remark 2: If the feedback $\alpha(x)$ satisfying (26) for $\mathcal{N}=\mathcal{N}^{*}$ is unique (or equivalently, if $v$ in (27) is void, i.e., $\widetilde{F}(\widetilde{x}, v)=$ $\widetilde{f}(\widetilde{x})$ ), then minimum phase reduces to the notion of minimum phase in [26]: $\dot{\widetilde{x}}=\widetilde{F}(\widetilde{x})$ is asymptotically stable.

Remark 3: For linear systems, minimum phase is equivalent to the requirement that the transmission zeros of $\Sigma$ are all in the open left half-plane.

As explained before, we will now address the inner-outer factorization problem for the general noninvertible case by considering the (singular) optimal control problem of infimizing the cost criterion for $\Sigma$

$$
J\left(x_{0}, u\right)=\frac{1}{2} \int_{0}^{\infty}\|y(t)\|^{2} d t, \quad x(0)=x_{0}
$$

over all locally integrable inputs $u$ such that (28) is defined (these inputs are called admissible) and $\lim _{t \rightarrow \infty} x(t)=0$. The main assumption concerning this optimal control problem throughout this section is as follows.

Assumption 3: $P^{+}\left(x_{0}\right):=\inf \left\{J\left(x_{0}, u\right): u\right.$ admissible such that $\left.\lim _{t \rightarrow \infty} x(t)=0\right\}$ exists for all $x_{0} \in \mathcal{M}$, and $P^{+}$is a $C^{\infty}$ function on $\mathcal{M}$.

Dynamic programming arguments (see, e.g., [45]) then imply that $P^{+}$is a solution to the integral dissipation inequality involving the unknown function $P: \mathcal{M} \rightarrow \mathbb{R}$

$$
\begin{aligned}
P\left(x\left(t_{1}\right)\right)-P\left(x\left(t_{0}\right)\right)+\frac{1}{2} \int_{t_{0}}^{t_{1}}\|y(t)\|^{2} d t & \geq 0 \\
P(0) & =0
\end{aligned}
$$

for all $t_{1} \geq t_{0}, x\left(t_{0}\right) \in \mathcal{M}$, and for all admissible inputs $u$ to $\Sigma$, with $x\left(t_{1}\right)$ the state at time $t_{1}$ resulting from state $x\left(t_{0}\right)$ at time time $t_{0}$ and the particular input function $u$. Since $P^{+}$is assumed to be differentiable, it is also a solution to the infinitesimal $\left(t_{1} \rightarrow\right.$ $t_{0}$ ) version of (29), the differential dissipation inequality

$$
\begin{aligned}
P_{x}(x) F(x, u)+\frac{1}{2} G(x, u)^{T} G(x, u) & \geq 0 \text { for all } x, u \\
P(0) & =0
\end{aligned}
$$

with $P_{x}(x)=\left(\partial P / \partial x_{1}(x), \ldots, \partial P / \partial x_{n}(x)\right)$ equal to the gradient vector of $P$ (written as a row vector). Note that any solution $P$ to (30) is also a solution to (29). In fact $P^{+}$is the maximal solution to either (29) or (30); see [41] for a similar argument in the linear case.

Remark 4: The assumption that $P^{+}$is smooth can be sought to be relaxed by using an appropriate notion of generalized solution of the Hamilton-Jacobi (30). The most natural notion of generalized solution in this control context appears to be the notion of viscosity-sense solution introduced by Crandall and Lions; see [12] for a comprehensive exposition. By working with this generalized notion of solution, most of the statements seem to carry over to a more natural level of generality. However, we shall henceforth deal only with smooth, classical solutions of (30).

Lemma 5: Let $P: \mathcal{M} \rightarrow \mathbb{R}$ satisfy (29), or let $P$ be $C^{1}$ and satisfy (30). Then, $P(x) \leq P^{+}(x)$ for $x \in \mathcal{M}$.

Proof: Consider (29) with $t_{0}=0$ for an admissible $u$ such that $\lim _{t \rightarrow \infty} x(t)=0$, and let $t_{1} \rightarrow \infty$. Then it follows that

$$
\frac{1}{2} \int_{0}^{\infty}\|y(t)\|^{2} d t \geq P(x(0))
$$

and thus by definition of $P^{+}$we obtain $P^{+}(x(0)) \geq P(x(0))$ for all $x(0) \in \mathcal{M}$.

Thus, $P^{+}$is completely characterized as the maximal solution to either (29) or (30), and, in principle, may be computed in this way.

Now, consider the smooth function

$$
K^{+}(x, u):=P_{x}^{+}(x) F(x, u)+\frac{1}{2} G^{T}(x, u) G(x, u) \geq 0 .
$$

Our next main assumption is as follows. 
Assumption 4: There exists a smooth mapping $\bar{G}: \mathcal{M} \times \mathbb{R}^{m} \rightarrow \mathbb{R}^{\bar{p}}$ for some $\bar{p}$, such that

$$
K^{+}(x, u)=\frac{1}{2} \bar{G}^{T}(x, u) \bar{G}(x, u) .
$$

Note that without the smoothness condition, Assumption 4 is trivially satisfied, since we may take $\bar{p}=1$ and $\bar{G}(x, u)=$ $\sqrt{K^{+}(x, u)}$. Sufficient conditions for the local existence of a smooth $\bar{G}$ satisfying (32) are provided by the next lemma which is a slight generalization of Morse's Lemma. For the proof, we refer to [27].

Lemma 6: Suppose that the Hessian matrix of $K^{+}$, i.e.,

$$
\left(\begin{array}{ll}
\frac{\partial^{2} K^{+}}{\partial x^{2}}(x, u) & \frac{\partial^{2} K^{+}}{\partial x \partial u}(x, u) \\
\frac{\partial^{2} K^{+}}{\partial u \partial x}(x, u) & \frac{\partial^{2} K^{+}}{\partial u^{2}}(x, u)
\end{array}\right)
$$

has constant rank, say $\bar{p}$, on a neighborhood of $(x, u)=(0,0)$. Then, locally near $(0,0)$ there exists a $C^{\infty}$ mapping $\bar{G}: \mathcal{M} \times$ $\mathbb{R}^{m} \rightarrow \mathbb{R}^{\bar{p}}$ such that (32) is satisfied.

Now, let us define the new system

$$
\bar{\Sigma}:\left\{\begin{array}{l}
\dot{x}=F(x, u) \\
\bar{y}=\bar{G}(x, u) .
\end{array}\right.
$$

If we consider the dissipation inequalities (29), (30) for $\bar{\Sigma}$, i.e.,

$$
\begin{aligned}
\bar{P}\left(x\left(t_{1}\right)\right)-\bar{P}\left(x\left(t_{0}\right)\right)+\frac{1}{2} \int_{t_{0}}^{t_{1}}\|\bar{y}(t)\|^{2} d t & \geq 0 \\
\bar{P}(0) & =0 \\
\bar{P}_{x}(x) F(x, u)+\frac{1}{2} \bar{G}^{T}(x, u) \bar{G}(x, u) & \geq 0 \\
\bar{P}(0) & =0
\end{aligned}
$$

we immediately arrive at the following.

Lemma 7: The maximal solution $\bar{P}^{+}$to either (34) or (35) is $\bar{P}^{+}=0$.

Proof: Clearly $\bar{P}=0$ satisfies (34) and (35). Now, let $\bar{P}$ satisfy, e.g., (35). Plug (31) and (32) into (35) to see that $P^{+}+\bar{P}$ is a solution to (30). Since $P^{+}$is maximal, this implies that $\bar{P} \leq 0$.

Since we have followed the general procedure in (22)-(25), we know that the system $\bar{\Sigma}$ given by (33) provides a stable solution of the spectral factorization problem (21). It remains to show that the special choice of $P$ as in Assumption 3 guarantees that $\bar{\Sigma}$ in addition has stable output-nulling dynamics. For this, we will impose some additional assumptions on the maximal solution $P^{+}$of (29) or (30) defined in Assumption 3. Note that by definition $P(x) \geq 0$ for all $x \in \mathcal{M}$, and $P^{+}(0)=0$. Therefore if $P^{+}$is positive definite around 0 , that is $P^{+}(x)>0$ for all $x \neq 0$ in a neighborhood of 0 , then we may immediately invoke standard Lyapunov theory to arrive at the following result.

Proposition 8: Given that Assumptions 1, 5, and 8 hold, let $P^{+}$be the maximal solution to (34), and let $\bar{\Sigma}$ be the associated system (37). Then, $\bar{\Sigma}$ is weakly minimum phase if $P^{+}$is positive definite around 0 .

Proof: We need to show: if $x \rightarrow \alpha(x)$ is output-nulling state feedback for $\Sigma$ (so $\bar{G}(x, \alpha(x))=0$ for all $x$ ) then the associated closed-loop dynamics $\dot{x}=F(x, \alpha(x))$ on $\mathcal{N}^{*}$ is stable w.r.t. $x=0$. From the factorization (36), we see that the closed-loop dynamics satisfies

$$
\begin{aligned}
\frac{d}{d t} P^{+}(x(t)) & =P_{x}^{+}(x(t)) F(x(t), \alpha(x(t))) \\
& =-\frac{1}{2} G^{T}(x, \alpha(x)) G(x, \alpha(x)) \leq 0
\end{aligned}
$$

showing stability of $x=0$ if $P^{+}$is positive-definite around 0 .

Remark 9: If additionally the largest invariant set within $\left\{x \in \mathcal{N}^{*} \mid G(x, \alpha(x))=0\right\}$ is $x=0$ then (36) implies that the closed-loop dynamics $\dot{x}=F(x, \alpha(x))$ on $\mathcal{N}^{*}$ is asymptotically stable, and thus $\bar{\Sigma}$ is minimum phase.

However, although $P^{+} \geq 0$ is the maximal solution to (34), it need not be positive definite in general. In fact, if $\Sigma$ itself is already minimum phase, then it directly follows from the definition of $P^{+}$in Assumption 5 that $P^{+}(x)=0$ for all $x$ in the maximal controlled invariant output-nulling submanifold of $\Sigma$ ! Therefore, in order to show minimum phase properties, we may as well start from Lemma 7, stating that

$$
\begin{aligned}
\bar{P}^{+}\left(x_{0}\right)=\inf \left\{\frac{1}{2} \int_{0}^{\infty}\|\bar{y}(t)\|^{2} d t ; u\right. \\
\left.\quad \text { admissible such that } \lim _{t \rightarrow \infty} x(t)=0\right\}
\end{aligned}
$$

is zero for all $x_{0} \in \mathcal{M}$. In fact, if we are allowed to replace in (37) "inf" by "min" for all $x_{0} \in \mathcal{N}^{*}$ then we directly conclude that $\bar{\Sigma}$ is minimum phase. (Since in this case there exists an admissible control $u$ such that $\|\bar{y}(t)\|=0, t \geq 0$, and $\lim _{t \rightarrow \infty} x(t)=$ 0.) The general case however remains somewhat elusive. For example, one may imagine that the output-nulling dynamics is partly unstable (in a nonlinear sense), while it may be possible to steer initial conditions $x_{0}$ in $\mathcal{N}^{*}$ to 0 while keeping $\|\bar{y}(t)\|$ arbitrarily small (implying that $\bar{P}^{+}\left(x_{0}\right)=0$ ). Hence, we conclude this section with a result based on the linearization of $\bar{\Sigma}$ and the linear theory developed in [17] and [41]. First, we consider for $\bar{\Sigma}$ the regularized cost criterion

$$
\frac{1}{2} \int_{0}^{\infty}\left(\|\bar{y}(t)\|^{2}+\epsilon^{2}\|u(t)\|^{2}\right) d t
$$

for $\epsilon$ small. This is a regular optimal control problem, and thus the maximal solution $\bar{P}_{\epsilon}^{+}$of the regularized dissipation inequality

$$
\bar{P}_{x}^{\epsilon}(x) F(x, u)+\frac{1}{2} \bar{G}^{T}(x, u) \cdot \bar{G}(x, u)+\frac{1}{2} \epsilon^{2} u^{T} u \geq 0
$$

actually is given as the stabilizing solution of the Hamilton-Jacobi-Bellman equation

$$
H_{\mathrm{opt}}^{\epsilon}\left(x, \bar{P}^{\epsilon^{T}}(x)\right)=0
$$

with $H_{\mathrm{opt}}^{\epsilon}(x, p):=H^{\epsilon}\left(x, p, u^{*}(x, p)\right)$, where $u^{*}(x, p)$ is the solution to $\partial H^{\epsilon} / \partial u(x, p, u)=0$, and

$$
H^{\epsilon}(x, p, u)=p^{T} F(x, u)+\frac{1}{2} \bar{G}^{T}(x, u) \bar{G}(x, u)+\frac{1}{2} \epsilon^{2} u^{T} u .
$$

If all data $F(x, u), \bar{G}(x, u)$ are smooth near 0 , then also $\bar{P}^{\epsilon}$ is a smooth function near $x=0$ (cf. [44]). It follows from the 
interpretation as an optimal control problem that $\bar{P}^{\epsilon} \geq 0$, and that $\bar{P}^{\epsilon}$ is nondecreasing as a function of $\epsilon$. Hence the pointwise limit $\bar{P}^{\lim }(x):=\lim _{\epsilon \downarrow 0} \bar{P}^{\epsilon}(x) \geq 0$ exists. We formulate the following theorem.

Theorem 10: Assume that the output-nulling dynamics of $\bar{\Sigma}$ does not have invariant eigenvalues on the imaginary axis (that is, it is hyperbolic). Furthermore, assume that $\bar{P}^{\lim }$ is a $C^{1}$ function. Then, $\bar{\Sigma}$ is minimum phase.

Proof: Suppose the output-nulling dynamics of $\bar{\Sigma}$ is not minimum phase. By the assumption of hyperbolicity this means that it is exponentially unstable. Thus for every smooth feedback $u=\alpha(x)$ such that the output

$$
\bar{y}=\bar{G}(\tilde{x}, \alpha(\tilde{x}))
$$

is identically zero along the closed-loop dynamics

$$
\dot{\tilde{x}}=F(\tilde{x}, \alpha(\tilde{x})), \quad \tilde{x} \in \mathcal{N}^{*}
$$

this closed-loop dynamics is exponentially unstable. Linearizing at $\tilde{x}=0$, this yields that for any such $\alpha, \exists \lambda \in \mathbb{C}$, $\operatorname{Re} \lambda>0$, and $\exists v \in \mathbb{C}^{n}, v \neq 0$, such that

$$
\begin{aligned}
& {\left[\frac{\partial F}{\partial x}(0,0)+\frac{\partial F}{\partial u}(0,0) \frac{\partial \alpha}{\partial x}(0)\right] v=\lambda v} \\
& {\left[\frac{\partial \bar{G}}{\partial x}(0,0)+\frac{\partial \bar{G}}{\partial u}(0,0) \frac{\partial \alpha}{\partial x}(0)\right] v=0 .}
\end{aligned}
$$

This implies that the linear system

$$
\begin{aligned}
& \dot{x}=\frac{\partial F}{\partial x}(0,0) x+\frac{\partial F}{\partial u}(0,0) u=: A x+B u \\
& \bar{y}=\frac{\partial \bar{G}}{\partial x}(0,0) x+\frac{\partial \bar{G}}{\partial u}(0,0) u=: C x+D u
\end{aligned}
$$

is not weakly minimum phase (transmission zeros in the open right half-plane). However by linear theory ([17], [41]) this means that there exists a solution $X=X^{T} \geq 0$, with $X \neq 0$, to the quadratic inequality corresponding to (39), i.e.,

$$
x^{T} X(A x+B u)+\frac{1}{2}[C x+D u]^{T}[C x+D u] \geq 0 .
$$

By continuity the regularized dissipation inequality (39) converges for $\epsilon \downarrow 0$ to

$$
\bar{P}_{x}^{\lim }(x) F(x, u)+\frac{1}{2} \bar{G}^{T}(x, u) \bar{G}(x, u) \geq 0
$$

and, hence, $\bar{P}^{\text {lim }}=\bar{P}^{+}=0$. On the other hand, by linearization at $x=0$

$$
\frac{\partial^{2} \bar{P}^{\epsilon}}{\partial x^{2}}(0) \geq X \neq 0
$$

for all $\epsilon>0$, which yields a contradiction.

The inner factor $\Theta$ is determined by $\Sigma=\Theta \circ \bar{\Sigma}$. Thus, if $\Sigma$ has input signals $u$ and output signals $y$ while $\bar{\Sigma}$ has $u$ as input and $\bar{y}$ as output, it must be that $\Theta$ has $\bar{y}$ as input and $y$ as output. This leads to the state-space representation for $\Theta$ with driving variable $u$

$$
\Theta:\left\{\begin{array}{l}
\dot{x}=F(x, u) \\
y=G(x, u) \\
\bar{y}=\bar{G}(x, u) .
\end{array}\right.
$$

Indeed, by considering (29) and (32) for $P=P^{+}$, we obtain

$$
\begin{aligned}
P^{+}\left(x\left(t_{1}\right)\right)-P^{+}\left(x\left(t_{0}\right)\right)+\frac{1}{2} \int_{t_{0}}^{t_{1}} & \|y(t)\|^{2} d t \\
& =\frac{1}{2} \int_{t_{0}}^{t_{1}}\|\bar{y}(t)\|^{2} d t
\end{aligned}
$$

which shows that $\Theta$ is lossless (from $\bar{y}$ to $y$ ) with storage function $P^{+}$. Note that the dynamics of $\Theta$ (more precisely, state trajectories $x(t)$ associated with an arbitrary initial condition $x(0)$ and zero input $\bar{y}=0$ ) is the same as the output-nulling dynamics for $\bar{\Sigma}$, and thus $\Theta$ is inner under the same hypotheses on $P^{+}$as was used to determine whether $\bar{\Sigma}$ is weakly minimum phase in Proposition 8 and Theorem 10. Note that if $\Sigma$ already happens to be minimum phase, then $P^{+}=0$ and the inner factor $\Theta$ may be taken to be the static identity mapping.

We next note as in [44] that the maximality property of $P^{+}$ in Lemma 5 implies that the minimum phase property may be expressed by a "minimal delaying action" (see [1] and [40] for the linear case).

Proposition 11: Among all systems

$$
\widetilde{\Sigma}\left\{\begin{array}{lll}
\dot{\tilde{x}}=\widetilde{F}(\widetilde{x}, \widetilde{u}), & \widetilde{F}(0,0)=0, & \widetilde{u} \in \mathbb{R}^{m} \\
\widetilde{y}=\widetilde{G}(\widetilde{x}, \widetilde{u}), & \widetilde{G}(0,0)=0, & \widetilde{y} \in \mathbb{R}^{\tilde{p}}
\end{array}\right.
$$

satisfying

$$
[D \widetilde{\Sigma}]^{T} \circ \widetilde{\Sigma}=[D \Sigma]^{T} \circ \Sigma
$$

as constructed by the procedure in (22)-(25), the minimal phase spectral factor $\bar{\Sigma}$ is characterized as that system $\bar{\Sigma}$ for which we have that, for $x(0)=0, \widetilde{x}(0)=0$ and for all $u=\widetilde{u}, T \geq 0$

$$
\int_{0}^{T}\|\bar{y}(t)\|^{2} d t \geq \int_{0}^{T}\|\widetilde{y}(t)\|^{2} d t .
$$

Proof: By the construction of stable spectral factors $\widetilde{\Sigma}$ in (22)-(25) we have $\widetilde{F}(x, u)=F(x, u)$ and that the outer factor has the form $\bar{\Sigma}$ as in (33) constructed from the solution $P^{+}$of (29) or (30) as in Assumption 3. Then driving either system $\widetilde{\Sigma}$ or $\bar{\Sigma}$ from rest $x(0)=\widetilde{x}(0)=0$ with the same input $u=\widetilde{u}$ results in $x(t)=\widetilde{x}(t)$ for all $t \geq 0$. Moreover, from (24) we have that the energy of the output $\|\widetilde{y}(t)\|^{2}$ is given by

$$
\begin{aligned}
\|\widetilde{y}(t)\|^{2}= & \widetilde{G}^{T}(x(t), u(t)) \widetilde{G}(x(t), u(t)) \\
= & \widetilde{P}_{x}(x(t)) F(x(t), u(t)) \\
& +\frac{1}{2} G^{T}(x(t), u(t)) G(x(t), u(t)) \\
= & \frac{d}{d t}\{\widetilde{P}(x(t))\}+\frac{1}{2} G^{T}(x(t), u(t)) G(x(t), u(t))
\end{aligned}
$$

for a solution $\widetilde{P}(x)$ of the dissipation inequality (23). Similarly

$$
\|y(t)\|^{2}=\frac{d}{d t}\left\{P^{+}(x(t))\right\}+\frac{1}{2} G^{T}(x(t), u(t)) G(x(t), u(t)) .
$$

Thus

$$
\begin{aligned}
& \int_{0}^{T}\|\bar{y}(t)\|^{2} d t-\int_{0}^{T}\|\widetilde{y}(t)\|^{2} d t \\
&=P^{+}(x(T))-\widetilde{P}(x(T)) \geq 0
\end{aligned}
$$


by the maximality property of $P^{+}$in Lemma 5 , and the result follows.

Remark 12: Note that the derivation of the "minimal delaying action" characterization of minimal-phase in Proposition 11 used only Assumptions 2 and 3, and did not require special assumptions on $P^{+}$used in Proposition 8 or Theorem 10. Also, the property of maximum phase can be similarly characterized by reversing the inequality in (47) under the analogous conditions.

Remark 13: (Control Problems With the Same Set of Optimal Controls) The conversion from $G$ to $\bar{G}$, cf. (31) and (32), and the resulting transformation of $\Sigma$ to $\bar{\Sigma}$ has a direct implication for the ability of transforming a nonlinear optimal control problem into another nonlinear optimal control problem with the same optimal controls. This observation generalizes some of the ideas exposed in Anderson [1] for linear systems to the nonlinear case. Consider the optimal control problem of minimizing $\int_{0}^{\infty} L(x, u) d t$ for the dynamics $\dot{x}=F(x, u)$, leading to the pre-Hamiltonian

$$
H(x, p, u)=p^{T} F(x, u)+L(x, u)
$$

where $L(x, u) \geq 0$ and $L(0,0)=0$. Consider now a different cost-criterion $\bar{L}(x, u), \bar{L}(x, u) \geq 0, \bar{L}(0,0)=0$ with regard to the same dynamics $\dot{x}=F(x, u)$, yielding the pre-Hamiltonian

$$
\bar{H}(x, \bar{p}, u)=\bar{p}^{T} F(x, u)+\bar{L}(x, u) .
$$

In general, the optimal controls and performances corresponding to $L$ and $\bar{L}$ are different. However, if there exists a canonical transformation $p=\bar{p}+R_{x}^{T}(x)$, for some function $R$, such that $H\left(x, \bar{p}+R_{x}^{T}(x), u\right)=\bar{H}(x, \bar{p}, u)$ or, equivalently

$$
R_{x}(x) F(x, u)+L(x, u)=\bar{L}(x, u) \text { for } \operatorname{all}(x, u)
$$

then the optimal controls for both optimal control problems are equal. The performance indices, however, denoted by $V$ and $\bar{V}$, respectively, are different, but are related via

$$
V(x)=\bar{V}(x)+R(x) .
$$

To see this, note that the equality $p=\bar{p}+R_{x}^{T}(x)$ implies that $V_{x}^{T}(x)=\bar{V}_{x}^{T}(x)+R_{x}^{T}(x)$.

If $L(x, u)=1 / 2\|G(x, u)\|^{2}$ and $\bar{L}(x, u)=1 / 2\|\bar{G}(x, u)\|^{2}$, then this is the situation studied before for inner-outer factorization. In this case, the analysis expresses the fact that the system $\Sigma$ is more difficult to control (has higher performance index) than its minimum phase factor $\bar{\Sigma}$.

\section{NONLINEAR SMITH PREDICTOR}

A useful property of the outer factor $\bar{\Sigma}$ as related to the original system $\Sigma$ is that $\bar{\Sigma}$ and $\Sigma$ have the same static gains, in the following sense, cf. [44]. Consider the set of all controlled equilibria, i.e.,

$$
\mathcal{E}=\left\{(x, u) \in \mathcal{M} \times \mathbb{R}^{m}: F(x, u)=0\right\} .
$$

Proposition 14: Consider $\Sigma$ and $\bar{\Sigma}$. For every $(x, u) \in \mathcal{E}$, we have

$$
\|G(x, u)\|=\|\bar{G}(x, u)\| .
$$

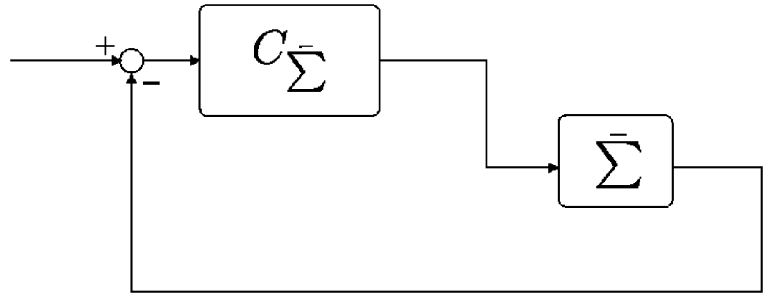

Fig. 1. Controller $C_{\sum}$ bases on outer factor $\bar{\sum}$.

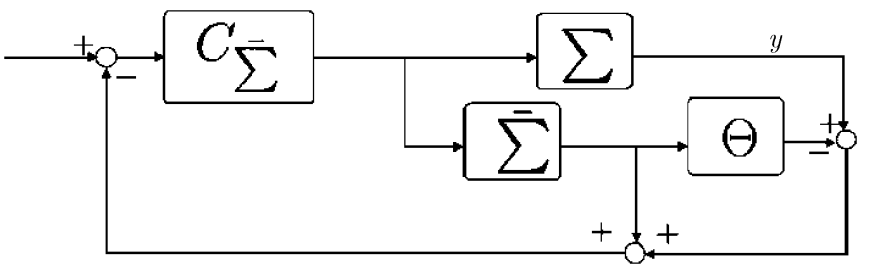

Fig. 2. Addition of compensating signal flows.

Proof: Simply consider the equality

$$
P_{x}^{+}(x) F(x, u)+\frac{1}{2} G^{T}(x, u) G(x, u)=\frac{1}{2} \bar{G}^{T}(x, u) \bar{G}(x, u)
$$

on $\mathcal{E}$.

This has the important consequence that if we compare the step responses of $\Sigma$ and $\bar{\Sigma}$ for every constant $u$, then the static gains of $\Sigma$ and $\bar{\Sigma}$ (assuming that the corresponding equilibrium $(x, u)$ of $\dot{x}=F(x, u)$ is (globally) asymptotically stable) are equal. Thus, for output set-point control of $\Sigma$ one may also consider its minimum phase factor $\bar{\Sigma}$, which is asymptotically based on $\bar{\Sigma}$, and since $\bar{\Sigma}$ is minimum phase, inversion techniques can be used. This idea, which generalizes an old idea in linear control theory (see, e.g., [35]), has been explored before in [49] and [16].

In this subsection we will show how in fact we can derive a nonlinear version of the structure of the classical Smith predictor (see e.g., [34] and [35]), based on the factorization $\Sigma=\Theta \circ \bar{\Sigma}$. This means that any controller $C_{\bar{\Sigma}}$ for the outer factor $\bar{\Sigma}$ will define a controller $C$ for the original system $\Sigma$.

Indeed, let us consider the plant system $\Sigma$ and its outer factor $\bar{\Sigma}$. Suppose that we have constructed for $\bar{\Sigma}$ a controller system $C_{\bar{\Sigma}}$ (e.g., by means of nonlinear inversion techniques such as nonlinear input-output decoupling, [25], [36]). Thus, we have the closed-loop system as depicted in Fig. 1. How do we derive from this configuration a controller for the original system $\Sigma$ ? We use the following argument stemming from the derivation of the classical Smith predictor. ${ }^{1}$ We add to the configuration of Fig. 1 two additional signal flows which exactly compensate each other, leading to Fig. 2

Subsequently, we shift the signal flow of $y$ to the left-hand side of the block diagram, in order to obtain Fig. 3.

The system within the dotted lines is now seen to be a controller for the original system $\Sigma$. We call it the nonlinear Smith predictor (based on the controller $C_{\bar{\Sigma}}$ for the outer factor of $\Sigma$ ).

Here, we shall not endeavor on an analysis of the properties of the nonlinear Smith predictor. Instead we refer to the analysis

\footnotetext{
${ }^{1}$ The third author would like to thank G. Meinsma for an illuminating discussion on the derivation of the Smith predictor.
} 


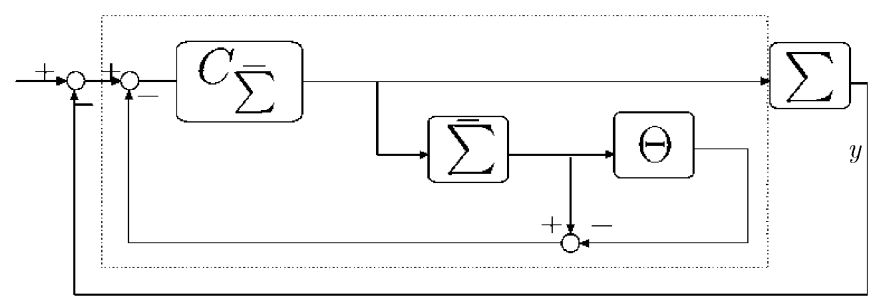

Fig. 3. Nonlinear smith predictor.

of controllers based on Smith predictors in the linear case, see, e.g., [35], and leave the interesting extension of these ideas to the nonlinear case for future research.

Note that the previous construction of the nonlinear Smith predictor may lead to problems in case $\Sigma$ is not a stable system (contrary to the standing assumption of this paper). Indeed, in this case the transition from Fig. 1 to Fig. 2 may lead to diverging signal behavior, in the sense that although in the complete absence of disturbances the two added signal flows exactly compensate each other, any disturbance or mismatch may easily lead to instabilities. (In the linear case, this is handled by the introduction of the modified Smith predictor; see, e.g., [34].)

\section{CONCLUSION}

In this paper, we have given a solution to the problem of inner-outer factorization for smooth nonlinear systems, extending the solution given before in the invertible case. Basically the extension entails the transition from the stabilizing solution of a Hamilton-Jacobi equation to the maximal solution of a Hamilton-Jacobi inequality, much in line with the state-space theory for inner-outer factorization of linear systems and the theory of dissipative systems [47], [48]. Finally, the potential of the obtained results for control of nonminimum phase nonlinear systems has been indicated; in particular we have derived a nonlinear Smith predictor based on the inner-outer factorization.

\section{REFERENCES}

[1] B. D. O. Anderson, "Algebraic properties of minimal degree spectral factors," Automatica, vol. 9, pp. 491-500, 1973.

[2] J. A. Ball, "Lossless dynamical systems and nonlinear Livsic-Brodskii nodes," in Operator Theory: Advances and Applications. Basel, Germany: Springer-Verlag, 1994, vol. 73.

[3] J. A. Ball and J. W. Helton, "Shift invariant manifolds and nonlinear analytic function theory," Int. Equat. Oper. Theory, vol. 11, pp. 615-725, 1988.

[4] — , "Factorization and general properties of nonlinear Toeplitz operators," in Operator Theory: Advances and Applications. Basel, Germany: Springer-Verlag, 1989, vol. 41.

[5] - "Inner-outer factorization of nonlinear operators," J. Function. Anal., vol. 104, pp. 363-413, 1992

[6] _ "Nonlinear $H_{\infty}$ control theory for stable plants," J. Math. Control, Signals, Syst., vol. 5, pp. 233-261, 1992.

[7] J. A. Ball, J. W. Helton, and M. Verma, "A factorization principle for stabilization of linear control systems," Int. J. Robust Nonlinear Control, vol. 1, pp. 229-294, 1991

[8] J. A. Ball and M. A. Petersen, "Nonlinear minimal square spectral factorization," Int. J. Control, 2004, to be published.

[9] J. A. Ball and M. Verma, "Factorization and feedback stabilization for nonlinear systems," Syst. Control Lett., vol. 23, pp. 187-196, 1991.

[10] J. A. Ball and A. J. van der Schaft, "Inner-outer factorization of nonlinear state space systems," in Systems and Networks: Mathematical Theory and Applications. Berlin, Germany: Springer-Verlag, 1994, vol. II, pp. 529-532.
[11] _ " " $J$-inner-outer factorization, $J$-spectral factorization and robust control for nonlinear systems," IEEE Trans. Automat. Contr., vol. 41, pp. 379-392, Mar. 1996.

[12] M. Bardi and I. Capuzzo-Dolcetta, Optimal Control and Viscosity Solutions of Hamilton-Jacobi-Bellman Equations. Boston, MA: Birkhäuser, 1997.

[13] C. I. Byrnes and A. Isidori, "Global feedback stabilization of nonlinear systems," in Proc. IEEE Conf. Decision Control, vol. 1031, Ft. Lauderdale, FL, 1985

[14] —_ "Asymptotic stabilization of minimum phase nonlinear systems," Syst. Control Lett., vol. 11, pp. 9-17, 1988.

[15] P. E. Crouch and A. J. van der Schaft, Variational and Hamiltonian Control Systems. Berlin, Germany: Springer-Verlag, 1987, Lecture Notes on Control and Information Science.

[16] F. J. Doyle, F. Allgöwer, and M. Morari, "A normal form approach to approximate input-output linearization for maximum phase nonlinear systems," IEEE Trans. Automat. Contr, vol. 41, pp. 305-309, Feb. 1996.

[17] B. A. Francis, "The optimal linear quadratic time-invariant regulator with cheap control," IEEE Trans. Automat. Contr., vol. AC-24, pp. 616-621, June 1979.

[18] P. A. Fuhrmann, "On the characterization and parametrization of minimal spectral factors," J. Math. Syst., Estimat., Control, vol. 5, pp. 383-444, 1995.

[19] P. A. Fuhrmann and A. Gombani, "On a hardy space approach to the analysis of spectral factors," Int. J. Control, vol. 71, pp. 277-357, 1998.

[20] — - "On the Lyapunov equation, coinvariant subspaces and some problems related to spectral factorizations," Int. J. Control, vol. 73, pp. $1129-1159,2000$

[21] T. Fujii, "On the perfect regulation of optimal regulators," Syst. Control Lett., vol. 1, pp. 356-359, 1982.

[22] M. Green, " $H_{\infty}$-controller synthesis by $J$-lossless coprime factorization," SIAM J. Control Optim., vol. 30, pp. 522-547, 1992.

[23] M. Green, K. Glover, D. J. N. Limebeer, and J. C. Doyle, "A $J$-spectral factorization approach to $H_{\infty}$-control," SIAM J. Control Optim., vol. 28, pp. 1350-1371, 1992.

[24] V. Ionescu and C. Oara, "Spectral and inner-outer factorizations for discrete-time systems," IEEE Trans. Automat. Contr., vol. 41, pp. 1840-1845, Nov. 1996.

[25] A. Isidori, Nonlinear Control Systems, 2nd ed. New York: SpringerVerlag, 1989

[26] A. Isidori and C. H. Moog, "On the nonlinear equivalent of the notion of transmission zeros," in Modeling and Adaptive Control, C. I. Byrnes and A. Kurzhanski, Eds. New York: Springer-Verlag, 1988, vol. 105, Lecture Notes in Control and Information Sciences, pp. 146-158.

[27] H. The. Jongen, P. Jonker, and F. Twilt, "Nonlinear optimization in finite dimensions: Morse theory, Chebyshev approximation, transversality, flows, parametric aspects," in Nonconvex Optimization and its Applications. Dordrecht, The Netherlands: Kluwer, 2000, vol. 47.

[28] H. Kimura, "Chain-scattering representation $J$-lossless factorization and $H_{\infty}$-control," J. Math. Syst., Estimat. Control, vol. 4, pp. 401-450, 1994.

[29] R. Kondo and S. Hara, "On cancellation in $H_{\infty}$ optimal controllers," Syst. Control Lett., vol. 13, pp. 205-210, 1989.

[30] C. Kravaris, "Input/output linearization: A nonlinear analog of placing poles at the process zeros," Amer. Inst. Chem. Eng. J., vol. 34, p. 1803, 1988.

[31] C. Kravaris and C. B. Chung, "Nonlinear feedback synthesis by global input/output linearization," Amer. Inst. Chem. Eng. J., vol. 33, p. 592, 1987.

[32] C. Kravaris and P. Daoutidis, "Nonlinear state space feedback control of second-order nonmiminum-phase nonlinear systems," Comput. Chem. Eng, vol. 14, pp. 439-449, 1990.

[33] H. Kwakernaak and R. Sivan, "The maximally achievable accuracy of linear optimal regulators and linear optimal filters," IEEE Trans. Automat. Contr., vol. AC-17, pp. 79-86, Jan. 1972.

[34] G. Meinsma and H. Zwart, "On $H_{\infty}$ control for dead-time systems," IEEE Trans. Automat. Contr., vol. 45, pp. 272-285, Feb. 2000.

[35] M. Morari and E. Zafiriou, Robust Process Control. Upper Saddle River, NJ: Prentice-Hall, 1989.

[36] H. Nijmeijer and A. J. van der Schaft, Nonlinear Dynamical Control Systems. New York: Springer-Verlag, 1990.

[37] M. A. Petersen and A. J. van der Schaft, "On a connection between nonlinear nonsquare spectral factors and Hamilton-Jacobi equations," in Nonlinear Control Systems, A. B. Kurzhanski and A. L. Fradkov, Eds. New York: Elsevier, 2002, vol. 3, pp. 1493-1498.

[38] _- "On nonlinear inner systems and connections with control theory," in Proc. 15th Triennial World Congr. International Federation of Automatic Control, Barcelona, Spain, July 21-26, 2002, p. 1656. 
[39] - Nonlinear Minimal Nonsquare Spectral Factorization, 2004, submitted for publication.

[40] E. A. Robinson, "Extremal representation of stationary stochastic processes," Ark. Mat., vol. 4, pp. 379-384, 1962.

[41] J. M. Schumacher, "The role of the dissipation matrix in singular optimal control," Syst. Control Lett., vol. 2, pp. 262-266, 1983.

[42] M. C. Tsai and I. Postlethwaite, "On $J$-lossless coprime factorizations and $H_{\infty}$ control," Int. J. Robust Nonlinear Control, vol. 1, pp. 47-68, 1991.

[43] A. J. van der Schaft, " $L_{2}$-gain analysis of nonlinear systems and nonlinear state feedback $H_{\infty}$-control," IEEE Trans. Automat. Contr., vol. 37, pp. 770-784, May 1992.

[44] —,$L_{2}$-gain and Passivity Techniques in Nonlinear Control, 2nd ed. London, U.K.: Springer-Verlag, 2000.

[45] E. D. Sontag, Mathematical Control Theory: Deterministic Finite Dimensional Systems, 2nd ed. New York: Springer-Verlag, 1998.

[46] A. Varga, "Computation of inner-outer factorizations of rational matrices," IEEE Trans. Automat. Contr., vol. 43, pp. 684-688, May 1998.

[47] J. C. Willems, "Least squares stationary optimal control and the algebraic Riccati equation," IEEE Trans. Automat. Contr., vol. AC-16, pp. 621-634, June 1971

[48] — - "Dissipative dynamical systems, Part I: General theory," Arch. Rat. Mech. Anal., vol. 45, pp. 321-351, 1972.

[49] R. A. Wright and C. Kravaris, "Nonminimum-phase compensation for nonlinear processes," Amer. Inst. Chem. Eng. J., vol. 38, pp. 26-40, 1992.

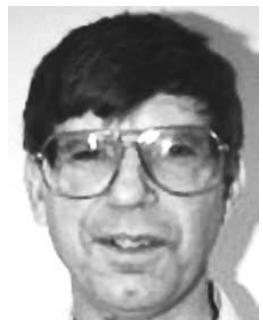

Joseph A. Ball was born in Washington, DC, in 1947. He received the B.S. degree in mathematics from Georgetown University, Washington, DC, in 1969, and the M.S. and Ph.D. degrees in mathematics from the University of Virginia, Charlottesville, in 1970 and 1973, respectively.

He joined the Faculty at Virginia Tech, Blacksburg, in 1973, where he is currently a Professor of mathematics. Short term visits elsewhere include the University of California at San Diego, the Weizmann Institute of Science, Rehovoth, Israel, the University of Maryland, College Park, the Vrije Universiteit, Amsterdam, The Netherlands, the Mathematical Sciences Research Institute, Berkeley, CA, and the Mittag-Lelfler Institute, Djursholm, Sweden. His interests include operator and function theory and its applications to control and system theory. He is currently an Associate Editor for Integral Equations and Operator Theory, the Journal of Mathematical Analysis and Applications, and Proceedings of the American Mathematical Society.

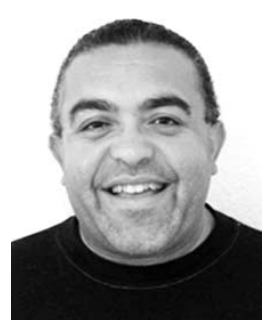

Mark A. Peterson was born in Elsies River, Cape Town, South Africa. He received the Ph.D. degree from the University of the Western Cape, Bellville, South Africa, in 1998.

His research interests are in factorization problems for nonlinear control systems and matrix functions and stochastic control in financial systems. He is currently a Professor of mathematics at the North West University, Potchefstroom, South Africa.

Dr. Peterson is a Member of the South African Mathematical Society (SAMS), the South African Council for Automation and Computation (SACAC), and serves on the Technical Committee for Economic Systems of the International Federation on Automatic Control (IFAC).

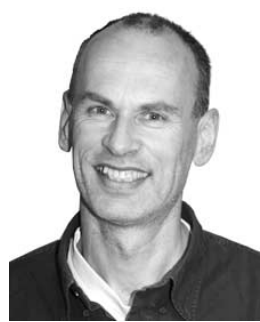

Arjan van der Schaft received the undergraduate and $\mathrm{Ph} . \mathrm{D}$. degrees in mathematics from the University of Groningen, Groningen, The Netherlands, in 1979 and 1983, respectively.

In 1982, he joined the Department of Applied Mathematics, University of Twente, Enschede, The Netherlands, where he is currently a Full Professor in mathematical systems and control theory. His research interests include the mathematical modeling of physical and engineering systems and the control of nonlinear and hybrid systems. He is the coauthor of the following books: System Theoretic Descriptions of Physical Systems (Amsterdam, The Netherlands: CWI, 1984), Variational and Hamiltonian Control Systems (New York: Springer-Verlag, 1987, with P.E. Crouch), Nonlinear Dynamical Control Systems (New York: Springer-Verlag, 1990, with $\mathrm{H}$. Nijmeijer), $L_{2}$-Gain and Passivity Techniques in Nonlinear Control (2nd edition, New York: Springer-Verlag, 2000), An Introduction to Hybrid Dynamical Systems (New York: Springer-Verlag, LNCIS 251, 2000, with J.M. Schumacher).

Dr. van der Schaft has served as Associate Editor for Systems and Control Letters, the Journal of Nonlinear Science, SIAM Journal on Control, and the IEEE TRANSACTIONS ON AUTOMATIC CONTROL. Currently, he is Associate Editor for Systems and Control Letters and Editor-at-Large for the European Journal of Control. 